\title{
Recent $B$ physics anomalies: a first hint for compositeness?
}

\author{
Adrián Carmona ${ }^{1,2, a}$, Florian Goertz ${ }^{3, b}$ \\ ${ }^{1}$ PRISMA Cluster of Excellence and Mainz Institute for Theoretical Physics, Johannes Gutenberg University, 55099 Mainz, Germany \\ 2 Theoretical Physics Department, CERN, 1 Esplanade des Particules, 1211 Geneva 23, Switzerland \\ ${ }^{3}$ Max-Planck-Institut für Kernphysik, Saupfercheckweg 1, 69117 Heidelberg, Germany
}

Received: 5 September 2018 / Accepted: 10 November 2018 / Published online: 26 November 2018

(C) The Author(s) 2018

\begin{abstract}
We scrutinize the recently further strengthened hints for new physics in semileptonic $B$-meson decays, focusing on the 'clean' ratios of branching fractions $R_{K}$ and $R_{K^{*}}$ and examining to which pattern of new effects they point to. We explore in particular the hardly considered, yet fully viable, option of new physics in the right-handed electron sector and demonstrate how a recently proposed framework of leptons in composite Higgs setups naturally solves both the $R_{K}$ and $R_{K^{*}}$ anomalies via a peculiar structure of new physics effects, predicted by minimality of the model and the scale of neutrino masses. Finally, we also take into account further observables, such as $\mathcal{B}\left(B_{s} \rightarrow \mu^{+} \mu^{-}\right), \Delta M_{B_{s}}$, and angular observables in $B \rightarrow K^{*} \mu^{+} \mu^{-}$decays, to arrive at a comprehensive picture of the model concerning (semileptonic) $B$ decays. We conclude that - since it is in good agreement with the experimental situation in flavor physics and also allows to avoid ultra-light top partners - the model furnishes a very promising scenarios of Higgs compositeness in the light of LHC data.
\end{abstract}

\section{Introduction}

Decays of $B$ mesons offer a promising place to search for new physics (NP), since in the Standard Model (SM) of Particle Physics flavor changing neutral processes are strongly suppressed and thus effects of NP might be sizable in (flavorchanging) $B$ decays. The case is strengthened by the fact that the bottom quark is the heaviest down-type quark and resides in the same weak doublet as the $t_{L}$, which due to its large mass is thought to furnish a major link to the completion of the SM at smallest distances.

\section{CERN-TH-2017-257, MITP/17-095.}

\footnotetext{
a e-mail: adrian.carmona@uni-mainz.de

b e-mail: fgoertz@mpi-hd.mpg.de
}

In fact, several anomalies have been found in $b \rightarrow s \ell^{+} \ell^{-}$ transitions, such as the long-standing anomaly in the angular analysis of the $B \rightarrow K^{*} \mu^{+} \mu^{-}$decay [1-5], as well as deficits in the branching fractions $B \rightarrow K \mu^{+} \mu^{-}$[6] and $B_{s} \rightarrow \phi \mu^{+} \mu^{-}$[7]. While these anomalies might be interpreted as a sign of new physics, ${ }^{1}$ some caution is in order because potentially sizable hadronic uncertainties are challenging to control.

A much cleaner probe of NP is given by ratios of branching fractions, like

$R_{K} \equiv \frac{\mathcal{B}\left(B^{+} \rightarrow K^{+} \mu^{+} \mu^{-}\right)}{\mathcal{B}\left(B^{+} \rightarrow K^{+} e^{+} e^{-}\right)}$

which tests lepton flavor universality (LFU), and where large hadronic uncertainties basically drop out [17] (see below). Interestingly, this theoretically very clean observable has also been measured at LHCb [18] and exhibits a sizable (25\%) depletion with respect to the SM prediction $[17,19]$ $\left|R_{K}^{\mathrm{SM}}-1\right|<1 \%$ in the $q^{2} \equiv\left(p_{\ell^{-}}+p_{\ell^{+}}\right)^{2} \in[1,6] \mathrm{GeV}^{2}$ bin, i.e.,

$\left.R_{K}^{[1,6] \exp } \equiv R_{K}\right|_{q^{2} \in[1,6] \mathrm{GeV}^{2}} ^{\exp }=0.745_{-0.074}^{+0.090} \pm 0.036$.

This corresponds to a deviation of almost $3 \sigma$. This intriguing hint for NP was recently strengthened with the measurement of the ratio

$R_{K^{*}} \equiv \frac{\mathcal{B}\left(B^{0} \rightarrow K^{*} \mu^{+} \mu^{-}\right)}{\mathcal{B}\left(B^{0} \rightarrow K^{*} e^{+} e^{-}\right)}$

which was found to be low by a similar amount [20]

$$
R_{K^{*}}^{[1.1,6] \exp }=0.69_{-0.07}^{+0.11} \pm 0.05
$$

\footnotetext{
${ }^{1}$ See [8-16] for theoretical interpretations in the framework of effective field theory.

2 This is valid above the muon threshold, $q^{2} \sim 4 m_{\mu}^{2}$.
} 
$R_{K^{*}}^{[0.045,1.1] \exp }=0.66_{-0.07}^{+0.11} \pm 0.03$,

where again $\left|R_{K^{*}}^{[1.1,6] \mathrm{SM}}-1\right|<1 \%$ and $\mid R_{K^{*}}^{[0.045,1.1] \mathrm{SM}_{-}}-\lesssim$ $5 \%$, resulting in tensions of $2.5 \sigma$ and $2.2 \sigma$, respectively. A naive combination of both results leads to a discrepancy with the SM of about $4 \sigma$, employing only observables that are theoretically well under control. ${ }^{3}$

In this article, we will focus on these clean observables, and study first the structure of NP required to explain the found deviations in an effective field theory (EFT) approach. We will in particular stress a solution with sizable effects in the right-handed electron sector, complementary to the common solution which is linked to the left-handed muon sector. We will then show how a recently proposed composite Higgs model, incorporating a non-trivial, yet minimal, implementation of the lepton sector $[21,22]$ can explain the $R_{K}$ and $R_{K^{*}}$ anomalies simultaneously, due to the peculiar chirality structure of the involved currents. The setup contains less degrees of freedom than standard realizations and is very predictive, leading in general to a non-negligible violation of LFU, while allowing at the same time for a strong suppression of flavor changing neutral currents (FCNCs). ${ }^{4}$

We will also discuss predictions of the setup for less clean observables in $b \rightarrow s \ell^{+} \ell^{-}$decays and take into account further flavor constrains on the model. We will in particular focus on the angular analysis of the $B \rightarrow K^{*} \mu^{+} \mu^{-}$decay, where pseudo-observables have been defined that also allow to cancel leading hadronic uncertainties [23-31], and where results are available from all 3 LHC $p p$ experiments as well as from Belle, which are again pointing to a $\sim 4 \sigma$ deviation from the SM. It turns out that non-negligible effects are also predicted in this decay, allowing a significant improvement with respect to the $\mathrm{SM}$, while still addressing the $R_{K^{(*)}}$ anomalies and meeting the most stringent flavor bounds.

The remainder of this article is organized as follows. In the next section, we will provide an analysis of the NP required to address the anomalies in LFU violating decays in terms of $D=6$ operators, parametrizing heavy physics beyond the SM in a model-independent way. We will then examine the structure of operators generated by the composite lepton model and present numerical predictions for $R_{K^{*}}$, scrutinizing the correlation with $R_{K}$ as well as $B_{S}-\bar{B}_{S}$ mixing and taking into account constraints from $B_{S} \rightarrow \mu^{+} \mu^{-}$. Finally, we will give our predictions for the angular observable $P_{5}^{\prime}$,

\footnotetext{
${ }^{3}$ Note that in the case of $R_{K}$ this holds also in the presence of NP, while for $R_{K^{*}}$ it holds within the SM, still allowing for a clean test of the latter.

4 Beyond that, in these models the leptonic contribution to the Higgs mass is parametrically enhanced relative to the quark contribution by (inverse) powers of $S O(5)$ breaking spurions, such that a light Higgs does not necessarily lead to light top partners, resolving tensions with LHC searches.
}

confronting them with the experimental results, before ending with our conclusions.

\section{Pattern of the $R_{K}, R_{K^{*}}$ anomalies}

Employing the framework of effective field theory, a clear picture of the required structure of heavy NP to explain the $R_{K^{(*)}}$ anomalies can be obtained. The relevant operators $\mathcal{O}_{i}$, contributing to semi-leptonic $B_{s}$ decays to leading approximation, are contained in the effective Hamiltonian $[32,33]$

$\mathcal{H}_{\mathrm{eff}}^{\Delta B=1}=-\frac{4 G_{F}}{\sqrt{2}} V_{t b} V_{t s}^{*} \frac{\alpha}{4 \pi} \sum_{i ; \ell=e, \mu, \tau}\left(C_{i}^{\ell} \mathcal{O}_{i}^{\ell}+C_{i}^{\ell^{\prime}} \mathcal{O}_{i}^{\ell^{\prime}}\right)+$ h.c.,

and read

$\mathcal{O}_{7}=\frac{m_{b}}{e}\left(\bar{s} \sigma_{\mu \nu} P_{R} b\right) F^{\mu \nu}, \quad \mathcal{O}_{9}^{\ell}=\left(\bar{s} \gamma_{\mu} P_{L} b\right)\left(\bar{\ell} \gamma^{\mu} \ell\right)$,

$\mathcal{O}_{10}^{\ell}=\left(\bar{s} \gamma_{\mu} P_{L} b\right)\left(\bar{\ell} \gamma^{\mu} \gamma_{5} \ell\right)$

and $\mathcal{O}_{7,9,10}^{\ell}{ }^{\prime}$ equivalently with $P_{L} \leftrightarrow P_{R}$, where $P_{L, R} \equiv$ $\left(1 \mp \gamma_{5}\right) / 2 .^{5}$ In fact, as the relevant energy scale is much below $m_{W}$, also the SM contributions are best expressed in terms of contributions to the operators (6), $C=C^{\mathrm{SM}}+C^{\mathrm{NP}}$ , where (at $\mu=4.8 \mathrm{GeV}$ ) [38]

$C_{9}^{\ell \mathrm{SM}}=4.07, C_{10}^{\ell \mathrm{SM}}=-4.31, C_{7}^{\mathrm{SM}}=-0.29$

respecting $\mathrm{LFU}$, i.e., $C_{i}^{e \mathrm{SM}}=C_{i}^{\mu \mathrm{SM}}=C_{i}^{\tau \mathrm{SM}}$, and $\mathcal{O}_{7,9,10}^{\ell}{ }^{\prime \text { SM }}=0$.

The operators above are written in terms of leptonic vector $\left(\mathcal{O}_{9}^{\ell^{(\prime)}}\right)$ and axial-vector $\left(\mathcal{O}_{10}^{\ell^{(\prime)}}\right)$ currents, which is convenient to add higher oder corrections including SM gauge bosons (the photon couples vectorial). NP, on the other hand, is conveniently parametrized in the chiral basis, writing the Hamiltonian (5) as

$$
\begin{aligned}
& \mathcal{H}_{\mathrm{eff}}^{\Delta B=1}=-\frac{4 G_{F}}{\sqrt{2}} V_{t b} V_{t s}^{*} \frac{\alpha}{4 \pi} \\
& \quad \times\left(\mathcal{O}_{7}+\mathcal{O}_{7}^{\prime}+\sum_{\substack{X, Y=L, R \\
\ell=e, \mu, \tau}} C_{b s_{X} \ell_{Y}} \mathcal{O}_{b s_{X} \ell_{Y}}\right)+\text { h.c. }
\end{aligned}
$$

\footnotetext{
5 The scalar and pseudo-scalar operators $\mathcal{O}_{S, P}^{\ell}{ }^{(\prime)}$ are already considerably constrained from $B_{s} \rightarrow \mu^{+} \mu^{-}$and play no important role in the following discussions [34,35] (for the electron channel, sizable effects are still possible in principle [36], however the operators play no role for our analysis of Sect. 3). This is also true for tensor operators, which can not be generated from operators invariant under the SM gauge group to leading order [37].
} 
with

$\mathcal{O}_{b s_{X} \ell_{Y}}=\left(\bar{s} \gamma_{\mu} P_{X} b\right)\left(\bar{\ell} \gamma^{\mu} P_{Y} \ell\right)$

since often NP models treat a certain chirality in a special way and one can take advantage of the accidental hierarchy of SM contributions

$C_{b s_{L} \ell_{L}}^{\mathrm{SM}}=8.38 \gg-C_{b s_{L} \ell_{R}}^{\mathrm{SM}}=0.24$

to directly see the importance of NP effects via their interference with the SM contributions (see $[39,40]$ ). The coefficients of the two bases are related in a simple way via $C_{9}^{\ell}=\left(C_{b s_{L} \ell_{R}}+C_{b s_{L} \ell_{L}}\right) / 2, C_{10}^{\ell}=\left(C_{b s_{L} \ell_{R}}-C_{b s_{L} \ell_{L}}\right) / 2$, and similarly for the primed operators with $b s_{L} \rightarrow b s_{R}$. In this basis, we arrive $\mathrm{at}^{6}$

$R_{K}=\frac{\left|C_{b s_{L} \mu_{L}}+C_{b s_{R} \mu_{L}}\right|^{2}+\left|C_{b s_{L} \mu_{R}}+C_{b s_{R} \mu_{R}}\right|^{2}}{\left|C_{b s_{L} e_{L}}+C_{b s_{R} e_{L}}\right|^{2}+\left|C_{b s_{L} e_{R}}+C_{b s_{R} e_{R}}\right|^{2}}$.

Given that we are considering corrections to $R_{K}$ of (up to) $\sim 30 \%$, the corresponding NP contributions to the Wilson coefficients will also be of this order, if they interfere with the leading SM contribution $C_{b s_{L} \ell_{L}}^{\mathrm{SM}}$ (barring significant cancellations). In consequence, it makes sense to expand $R_{K}$ to leading order in the NP coefficients $C_{b s_{X} \ell_{Y}}^{\mathrm{NP}}$, to allow for a transparent theoretical interpretation, which we will do below. We keep however terms containing right handed lepton currents up to quadratic order, going beyond the chiral linear approximation [40], since they do not interfere with the leading (left-handed) SM contribution (for vanishing lepton masses). Thus, on the one hand, potentially larger effects are required to explain the anomalies (as happens in the explicit model under consideration), and generically, due to the suppressed

${ }^{6}$ The individual branching fractions are given by $[13,17,39,41]$

$$
\begin{aligned}
\frac{d \mathcal{B}\left(B \rightarrow K \ell^{+} \ell^{-}\right)}{d q^{2}}= & \tau_{B^{+}} \frac{G_{F}^{2} \alpha^{2}\left|V_{t b} V_{t s}^{*}\right|^{2}}{(4 \pi)^{5} m_{B}^{3}}\left(\left[m_{B-K^{*}}^{2}\right]^{2}\right. \\
& \left.-2 m_{B+K^{*}}^{2} q^{2}+q^{4}\right)^{\frac{3}{2}}\left(\left|F_{V}\right|^{2}+\left|F_{A}\right|^{2}\right),
\end{aligned}
$$

where

$$
\begin{aligned}
F_{V}\left(q^{2}\right)= & \left(C_{b s_{R} \ell_{R}}+C_{b s_{L} \ell_{L}}+C_{b s_{R} \ell_{L}}+C_{b L_{L} \ell_{R}}\right) / 2 f_{+}\left(q^{2}\right) \\
& +\frac{2 m_{b}}{m_{B}+m_{K}}\left(C_{7}+C_{7}^{\prime}\right) f_{T}\left(q^{2}\right)+h_{K}\left(q^{2}\right), \\
F_{A}\left(q^{2}\right)= & \left(C_{b s_{R} \ell_{R}}-C_{b s L} \ell_{L}-C_{b s R} \ell_{L}+C_{b s L} \ell_{R}\right) / 2 f_{+}\left(q^{2}\right),
\end{aligned}
$$

$m_{A \pm B}^{2} \equiv m_{A}^{2} \pm m_{B}^{2}$, and we neglected lepton masses, CP violation, and higher order corrections (which are however included in our numerical analysis, employing $\mathrm{CP}$ averaged quantities). Here, $f_{+}$and $f_{T}$ are the QCD form factors (see $[42,43])$ and $h_{K}\left(q^{2}\right)$ parametrizes nonfactorizable contributions from the weak hamiltonian [13]. Neglecting the strongly suppressed $C_{7}^{(\prime)}$ contributions (which could only become relevant approaching the photon pole at $q^{2}=0$ and are in any case constrained to be pretty SM like [44]) and the non-factorizable $h_{K}\left(q^{2}\right)$, the QCD form factor $f_{+}\left(q^{2}\right)$ drops out in the ratio $R_{K}(13)$. interference, quadratic terms become important. Note however that (as long as $C_{b s_{X} \ell_{R}}^{\mathrm{NP}}<C_{b s_{L} \ell_{L}}^{\mathrm{SM}}$ ) higher terms are still suppressed. ${ }^{7}$ Neglecting also the strongly suppressed interference with $C_{b s_{L} \ell_{R}}^{\mathrm{SM}}$, we arrive at

$$
\begin{aligned}
R_{K} \simeq & 1+2 \frac{\operatorname{Re}\left[C_{b s_{L} \ell_{L}}^{\mathrm{SM} *}\left(C_{b s_{L} \mu_{L}}^{\mathrm{NP}}+C_{b s_{R} \mu_{L}}^{\mathrm{NP}}-C_{b s_{L} e_{L}}^{\mathrm{NP}}-C_{b s_{R} e_{L}}^{\mathrm{NP}}\right)\right]}{\left|C_{b s_{L} \ell_{L}}^{\mathrm{SM}}\right|^{2}} \\
& +\frac{\left|C_{b s_{L} \mu_{R}}^{\mathrm{NP}}+C_{b s_{R} \mu_{R}}^{\mathrm{NP}}\right|^{2}-\left|C_{b s_{L} e_{R}}^{\mathrm{NP}}+C_{b s_{R} e_{R}}^{\mathrm{NP}}\right|^{2}}{\left|C_{b s_{L} \ell_{L}}^{\mathrm{SM}}\right|^{2}}
\end{aligned}
$$

For generic $30 \%$ corrections to $R_{K}$, this formula is exact up to $\lesssim 10 \%$ corrections, which are smaller than the experimental uncertainty. ${ }^{8}$ In particular, it captures the leading effects of all NP Wilson coefficients considered.

We directly observe that $R_{K}<1$ can be realized in two ways. It could origin from a destructive (constructive) interference of the combined left-handed muon (electron) contributions with the leading SM piece or, more generally, a negative sign in the difference of muon and electron contributions $C_{b s_{L+R}(\mu-e)_{L}}^{\mathrm{NP}} \equiv C_{b s_{L} \mu_{L}}^{\mathrm{NP}}+C_{b s_{R} \mu_{L}}^{\mathrm{NP}}-C_{b s_{L} e_{L}}^{\mathrm{NP}}-C_{b s_{R} e_{L}}^{\mathrm{NP}}$. On the other hand, it could stem from couplings to right-handed lepton currents. In that case, as discussed, the quadratic NP contribution dominates in general. From (14) it then follows directly that $R_{K}<1$ requires the effect to come from the electron sector. Of course, a combination is possible, such that right-handed muon currents are allowed, however, while for the case of electron currents, any operator alone could accommodate $R_{K}<1$, for the muon case, right handed contributions alone are not feasible, no matter what is the quark chirality.

More valuable information on the physical origin of the possible $B$-physics anomalies can be obtained by considering in addition the ratio $R_{K^{*}}$, which tests different combinations

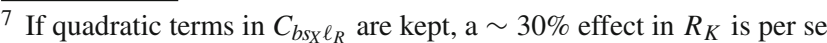
in agreement with a convergence of the expansion in NP contributions, no matter from which operator it is induced (see also [45]).

${ }^{8}$ If the fourth order in $C_{b s_{X}} \ell_{R}$ is included, which corresponds to adding

$\Delta R_{K}^{(4)}=\frac{\left|C_{b s_{L} e_{R}}^{\mathrm{NP}}+C_{b s_{R} e_{R}}^{\mathrm{NP}}\right|^{4}-\left|C_{b s_{L} e_{R}}^{\mathrm{NP}}+C_{b s_{R} e_{R}}^{\mathrm{NP}}\right|^{2}\left|C_{b s_{L} \mu_{R}}^{\mathrm{NP}}+C_{b s_{R} \mu_{R}}^{\mathrm{NP}}\right|^{2}}{\left|C_{b s_{L} \ell_{L}}^{\mathrm{SM}}\right|^{4}}$,
}

it holds even at the $\mathcal{O}(1 \%)$ level, which becomes negligible compared to other uncertainties. Still, for the numerical results presented in Sect. 3 , we will use the exact expressions, including in addition higher order QCD corrections [46] as well as the effect of $C_{7}^{\mathrm{SM}}$. 
of Wilson coefficients, to which we will turn now. The theoretical prediction in this case reads $(p \approx 0.86)^{9}$ unless both left- and right-handed quark currents appear in LFU violating NP contributions.

$$
R_{K^{*}}=\frac{(1-p)\left(\left|C_{b s_{L} \mu_{L}}+C_{b s_{R} \mu_{L}}\right|^{2}+\left|C_{b s_{L} \mu_{R}}+C_{b s_{R} \mu_{R}}\right|^{2}\right)+p\left(\left|C_{b s_{L} \mu_{L}}-C_{b s_{R} \mu_{L}}\right|^{2}+\left|C_{b s_{L} \mu_{R}}-C_{b s_{R} \mu_{R}}\right|^{2}\right)}{(1-p)\left(\left|C_{b s_{L} e_{L}}+C_{b s_{R} e_{L}}\right|^{2}+\left|C_{b s_{L} e_{R}}+C_{b s_{R} e_{R}}\right|^{2}\right)+p\left(\left|C_{b s_{L} e_{L}}-C_{b s_{R} e_{L}}\right|^{2}+\left|C_{b s_{L} e_{R}}-C_{b s_{R} e_{R}}\right|^{2}\right)}
$$

Expanding again in $C_{b s_{X} \ell_{Y}}^{\mathrm{NP}}$, keeping quadratic terms only in $C_{b s_{X} \ell_{R}}^{\mathrm{NP}}$ and neglecting the strongly suppressed interference with $C_{b s_{L} \ell_{R}}^{\mathrm{SM}}$, we obtain

$$
\begin{aligned}
R_{K^{*}} \simeq R_{K}-4 p \frac{\operatorname{Re}\left[C_{b s_{L} \ell_{L}}^{\mathrm{SM} *}\left(C_{b s_{R} \mu_{L}}^{\mathrm{NP}}-C_{b s_{R} e_{L}}^{\mathrm{NP}}\right)\right]}{\left|C_{b s_{L} \ell_{L}}^{\mathrm{SM}}\right|^{2}} \\
\\
-4 p \frac{\operatorname{Re}\left[C_{b s_{L} \mu_{R}}^{\mathrm{NP}} C_{b s_{R} \mu_{R}}^{\mathrm{NP}}-C_{b s_{L} e_{R}}^{\mathrm{NP}} C_{b s_{R} e_{R}}^{\mathrm{NP} *}\right]}{\left|C_{b s_{L} \ell_{L}}^{\mathrm{SM}}\right|^{2}} .
\end{aligned}
$$

From this expression it is evident that $R_{K} *$ probes righthanded quark currents since in their absence it becomes equivalent to $R_{K}$ to leading approximation. A similar conclusion holds in the absence of left-handed lepton currents,

9 Neglecting terms suppressed by $m_{\ell}^{2} / q^{2}$, NLO, and non-factorizable corrections (as well as CP violation), the individual branching fractions can be expressed in terms of six transversity amplitudes $A_{0, \perp, \|}^{L, R}$ as [47] (see also $[39,41,48,49]$ )

$$
\begin{aligned}
\frac{d \mathcal{B}\left(B^{0} \rightarrow K^{*} \ell^{+} \ell^{-}\right)}{d q^{2}} \simeq & \tau_{B^{0}} \frac{G_{F}^{2} \alpha^{2}\left|V_{t b} V_{t s}^{*}\right|^{2}}{3 \cdot(4 \pi)^{5} m_{B}^{3}}\left(\left[m_{B-K^{*}}^{2}\right]^{2}\right. \\
& \left.-2 m_{B+K^{*}}^{2} q^{2}+q^{4}\right)^{\frac{1}{2}} q^{2} \\
& \left(\left|A_{\perp}^{\ell L}\right|^{2}+\left|A_{\|}^{L}\right|^{2}+\left|A_{0}^{L}\right|^{2}+L \rightarrow R\right),
\end{aligned}
$$

$A_{\perp}^{\ell L, R}=+\sqrt{2} m_{B}\left(1-q^{2} / m_{B}^{2}\right)\left[C_{b s_{L} \ell_{L, R}}+C_{b s_{R} \ell_{L, R}}\right] \xi_{\perp}$,

$A_{\|}^{\ell L, R}=-\sqrt{2} m_{B}\left(1-q^{2} / m_{B}^{2}\right)\left[C_{b s_{L} \ell_{L, R}}-C_{b s_{R} \ell_{L, R}}\right] \xi_{\perp}$,

$A_{0}^{\ell L, R}=-\frac{m_{B}^{3}\left(1-q^{2} / m_{B}^{2}\right)^{2}}{2|q| m_{K^{*}}}\left[C_{b s_{L} \ell_{L, R}}-C_{b s_{R} \ell_{L, R}}\right] \xi_{\|}$,

where the form factors $\xi_{\perp, \|}$ are given, e.g., in Appendix E of [47]. We directly dropped electromagnetic dipole contributions, becoming important only for $q^{2} \rightarrow 0$, which would appear in the three square brackets above as $2 m_{b} m_{B} / q^{2}\left\{C_{7}+C_{7}^{\prime}, C_{7}-C_{7}^{\prime}, q^{2} / m_{B}^{2}\left(C_{7}-C_{7}^{\prime}\right)\right\}$. Defining the integrated form factors

$$
\begin{gathered}
g_{\perp, \|, 0}^{\left[q_{\min }^{2}, q_{\max }^{2}\right]}=\int_{q_{\min }^{2}}^{q_{\max }^{2}} d q^{2}\left(\left[m_{B-K^{*}}^{2}\right]^{2}-2 m_{B+K^{*}}^{2} q^{2}+q^{4}\right)^{\frac{1}{2}} \\
\times \frac{2\left(q^{3}-m_{B}^{2} q\right)^{2}}{m_{B}^{2}}\left\{\left|\xi_{\perp}\right|^{2},\left|\xi_{\perp}\right|^{2}, \frac{\left(m_{B}^{2}-q^{2}\right)^{2}}{8 q^{2} m_{K^{*}}^{2}}\left|\xi_{\|}\right|^{2}\right\},
\end{gathered}
$$

we can write $R_{K^{*}}$ as two combinations of Wilson coefficients, weighted by the polarization fraction $p \equiv \frac{g_{0}+g_{\|}}{g_{0}+g_{\|}+g_{\perp}}$, where $p \approx 0.86$ to good approximation for the $q^{2}$ range considered here (with some per cent deviation for the low $q^{2}$ bin) $[39,47]$.
The findings above are visualized in Fig. 1, where we show the correlations between $R_{K}$ and $R_{K^{*}}$, employing Eqs. (13) and (18). The colored lines correspond to the effects of the various NP Wilson coefficients. We consider all the coefficients entering these expressions, including combinations, such as to allow simultaneously for NP in the muon and electron sectors and in different chirality combinations. The most important dependencies of $R_{K}$ and $R_{K} *$ are on the difference of purely left-handed contributions involving muons and electrons $C_{b s_{L}(\mu-e)_{L}}^{\mathrm{NP}} \equiv C_{b s_{L} \mu_{L}}^{\mathrm{NP}}-C_{b s_{L} e_{L}}^{\mathrm{NP}}$, on the corresponding quantity involving right-handed quark currents $C_{b s_{R}(\mu-e)_{L}}^{\mathrm{NP}} \equiv C_{b s_{R} \mu_{L}}^{\mathrm{NP}}-C_{b s_{R} e_{L}}^{\mathrm{NP}}$, where the direction of positive values is indicated by an arrow, and on the four coefficients involving right handed lepton currents, entering at quadratic order in NP. Note that for the latter case, a simultaneous presence of left- and right-handed quark currents is necessary, in order to break the degeneracy $R_{K}=R_{K^{*}}$, while in case only one coefficient is turned on, the effect of either of them is indistinguishable in the $R_{K}$ vs. $R_{K}$ * plane. We thus consider the distinct individual contributions $C_{b s_{X} \mu_{R}}^{\mathrm{NP}}$ and $C_{b s_{X} e_{R}}^{\mathrm{NP}}$ (being equal for $X=L, R$ ) as well as the simultaneous presence of left- and right-handed quark currents via $C_{b s_{L} \ell_{R}}^{\mathrm{NP}}=C_{b s_{R} \ell_{R}}^{\mathrm{NP}} \equiv C_{b s_{(L=R)} \ell_{R}}^{\mathrm{NP}}$, to capture the most relevant different scenarios. The effect of further combining different contributions to $R_{K}$ and $R_{K^{*}}$ can be easily inferred by considering the analytic Eqs. (13) and (18) in addition to the figure. The size of the coefficients corresponding to a certain point in the plane is visualized via the shape of the lines - solid lines correspond to $0<\left|C_{b s_{X} \ell_{Y}}^{\mathrm{NP}}\right|<1$, dashed lines to $1<\left|C_{b s_{X} \ell_{Y}}^{\mathrm{NP}}\right|<2.5$, while dotted lines feature $2.5<\left|C_{b s_{X} \ell_{Y}}^{\mathrm{NP}}\right|<5$. Since the interference of NP effects in the right-handed lepton sector with SM contributions is suppressed, generically larger coefficients are required here in order to obtain sizable effects.

From the plot it becomes clear that the LHCb results, visualized by the black uncertainty bars, uniquely single out either a left-handed-left-handed NP effect, where $C_{b s_{L} \mu_{L}}^{\mathrm{NP}}\left(C_{b s_{L} e_{L}}^{\mathrm{NP}}\right)$ needs to feature a negative (positive) sign, or an effect in the right-handed electron sector via $C_{b s_{X} e_{R}}^{\mathrm{NP}}$, as the preferred solution to the $R_{K}^{(*)}$ anomalies. ${ }^{10}$ This is a very interesting finding

\footnotetext{
${ }^{10}$ It becomes also evident that a negative NP contribution to $C_{9}^{\mu}=$ $\left(C_{b_{L} \mu_{R}}+C_{b_{s_{L}} \mu_{L}}\right) / 2$, as advocated as a solution to the $B \rightarrow K^{*} \mu^{+} \mu^{-}$ anomaly (see, e.g., $[8,16])$, allows for a good fit of the $R_{K}^{(*)}$ anomalies, basically because (for moderate values of the coefficients) the effect of
} 

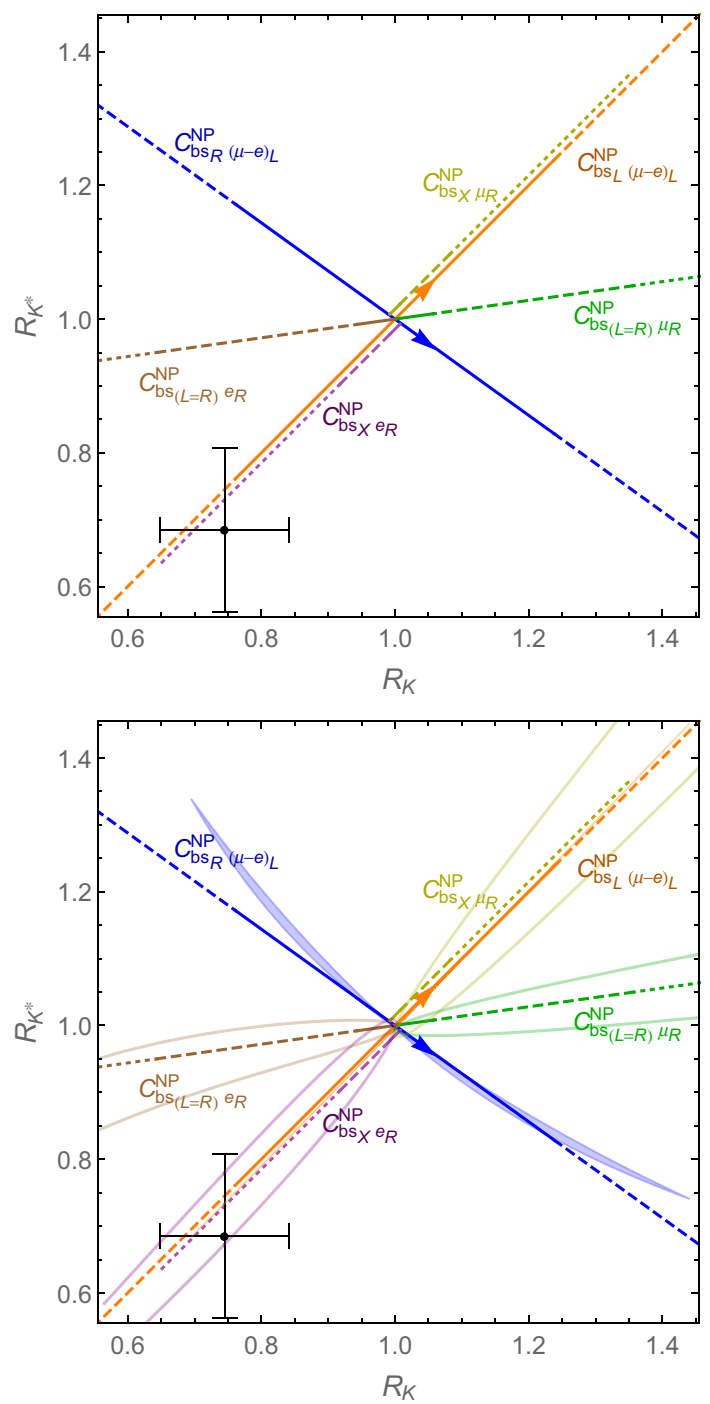

Fig. 1 Predictions for $R_{K}$ vs. $R_{K^{*}}$ in dependence on the values of the NP Wilson coefficients entering Eqs. (13) and (18), where solid, dashed, and dotted lines correspond to $0<\left|C_{b_{X} \ell_{Y}}^{\mathrm{NP}}\right|<1,1<\left|C_{b s_{X} \ell_{Y}}^{\mathrm{NP}}\right|<2.5$, and $2.5<\left|C_{b s_{X} \ell_{Y}}^{\mathrm{NP}}\right|<5$, respectively. The $1 \sigma$ allowed range from $\mathrm{LHCb}$ is depicted by the black uncertainty bars. Both anomalies can be addressed simultaneously via $\mathcal{O}_{b s_{X} e_{R}}$ or via $\mathcal{O}_{b s_{L}(\mu-e)_{L}}$. In the lower panel, the exact predictions are added as faint colored lines. See text for details

with respect to the model considered in the remainder of the paper. In fact, while a number of models accommodate the former option of dominating left-handed effects (including leptonic vector currents), see [50-73] for $Z^{\prime}$ realizations, the latter solution hardly exists in the form of explicit models in the literature, however just emerges in the composite model presented in Sect. 3.

Footnote 10 continued

$C_{b s_{L} \mu_{L}}^{\mathrm{NP}}$ dominates via the SM interference. A positive $C_{9}^{e}$, on the other hand, also allows for a straightforward solution.
We finally stress again that for the predictions of the model discussed in the next section, we employ full results including higher order corrections and the (small) effects of $C_{7}^{\mathrm{SM}}$, performing a quadratic fit in the NP Wilson coefficients to the NLO results. ${ }^{11}$ We also display, in the right panel of Fig. 1, the results employing these full expressions, visualized via faint colored lines. Note that now, in the case of right-left contributions, the prediction does not just depend on the difference of muon and electron contributions any more and varying $C_{b s_{R} \mu_{L}}^{\mathrm{NP}}$ and $C_{b s_{R} e_{L}}^{\mathrm{NP}}$ independently leads to a (very modest) spread of the predictions in the $R_{K}$ vs. $R_{K^{*}}$ plane, depicted by the blue shaded region. Generally, the approximate results describe the relevant physics quite accurately.

In summary, consistently explaining the $R_{K}$ and $R_{K^{*}}$ anomalies requires both quark FCNCs involving the $b$ quark and LFU violation in the $\mu$ vs. $e$ system (with effects either in left-handed currents in both sectors, or with a non-negligible right-handed electron contribution). The model that we will discuss now naturally leads to both effects, via exchange of composite vector resonance, whose couplings are not aligned with the SM couplings and the biggest contributions are actually expected in quark transitions involving the third generation and LFU violation involving light SM leptons. Since larger corrections are predicted for electrons, the setup matches nicely with the fact that this sector is less constrained, and, as we will see, sizable effects are in fact possible without problems with, e.g., $B_{S} \rightarrow \ell^{+} \ell^{-}$decays.

\section{Predictions in composite framework and further observables}

Composite Higgs models offer a priori a compelling framework to explain the neutral flavor anomalies. The presence of a rich spectrum of bound states at the $\mathrm{TeV}$ scale, including heavy vector resonances (of $Z^{\prime}$ type) with sizable couplings to some of the SM fermions, make these scenarios natural candidates to address the tension between data and SM predictions. Moreover, and contrary to most of the solutions to these anomalies that one can find in the literature, they also offer an interplay with electroweak symmetry breaking (EWSB) and some rationale to solve the hierarchy problem. If one considers that fermion masses are generated via the mechanism of partial compositeness, a sizable violation of lepton flavor universality, like suggested by $R_{K}$ and $R_{K^{*}}$, necessarily requires the charged leptons to feature a sizable degree of compositeness in their left-handed (LH) and/or right-handed (RH) chirality, $\epsilon_{\ell_{R}}, \epsilon_{\ell_{L}}$. Since charged lepton masses scale in general as $\sim g_{*} v \epsilon_{\ell_{L}} \epsilon_{\ell R}$, where $g_{*}$ is the characteristic coupling within the strong sector, both chiralities can not be composite at the same time. Therefore, these

${ }^{11}$ We used the code FLAVIO (v0.21.1) [46] for the numerical prediction. 
models will either predict effects in $\mathcal{O}_{b s_{L, R} \ell_{L}}$ or in $\mathcal{O}_{b s_{L, R} \ell_{R}}$ scaling like $\sim g_{*}^{2} / m_{*}^{2} V_{t s} \epsilon_{b_{X}}^{2} \epsilon_{\ell_{Y}}^{2}$, where $X$ and $Y$ denote the possible chiralities involved in the quark- and lepton-sector, respectively, and $m_{*}$ is the typical mass scale of the first vector resonances.

The model under consideration falls into the second category and was presented in detail in [21,22]. One of its most interesting features is that charged leptons partially substitute the role of the top quark as a trigger of EWSB, and a link between violation of lepton flavor universality and the absence of top partners at the LHC is established. Indeed, if the composite operators interacting with the $\mathrm{RH}$ charged leptons transform in sufficiently large irreducible representations of the global group within the strong sector, the leading charged lepton contribution to the Higgs quartic coupling will appear at order $\sim\left|\epsilon_{\ell_{R}}\right|^{2}$ instead of the usual $\sim\left|\epsilon_{\ell_{R}}\right|^{4}$. Since the leading top contribution can be expected to appear at order $\left|\epsilon_{t_{L}}\right|^{4}$, the contribution arising from the lepton sector can be comparable to the top one, even with a smaller degree of compositeness. Moreover, if all the three lepton generations are partially composite, the lepton contribution will be enhanced by a factor $N_{\text {gen }} \sim 3$, compensating the color factor $N_{c}=3$ present in the top case and allowing to lift the problematic top partners via destructive interference between the two sectors in the Higgs potential. ${ }^{12}$ One of the important findings of $[21,22]$ is that the very same representations making this possible also provide the required quantum numbers for a minimal implementation of a type-III seesaw mechanism for neutrino masses, which can in fact motivate RH charged-lepton compositeness, as we will see now. ${ }^{13}$ If one follows this very minimal avenue, considering each generation of $\mathrm{RH}$ leptons to interact with a single composite operator, all RH charged leptons in fact inherit the degree of compositeness of their RH neutrino counterparts, and the latter is required to be sizable to allow for large enough neutrino masses (see [21,22,77-80] for more details in both type-I and type-III seesaw models). Then, the different scaling of the neutrino and charged lepton mass matrices requires $0 \ll \epsilon_{\tau_{R}} \ll \epsilon_{\mu_{R}} \ll \epsilon_{e_{R}}$, in order to have simultaneously hierarchical charged lepton masses and a non-hierarchical neutrino mass matrix [21]. An immediate consequence of the above chirality structure is that mostly the operators $\mathcal{O}_{b_{\text {SX }} e_{R}}$ will be generated (as well as subdominantly $\left.\mathcal{O}_{b s X} \mu_{R}\right)$. Moreover, possible modifications of $Z$ couplings which are extremely constrained by electroweak precision data are avoided due to custodial symmetry, contrary to what happens for the case of composite LH leptons, where it is not possible to protect the coupling to both fields in the SM doublet at the same time.

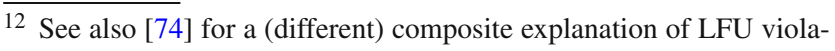
tion.

13 This is true at least for the $S O(5) / S O(4)$ and the $S O(7) / G_{2}$ [75] cosets (see also [76]).
}

To be concrete, we are considering a strongly interacting sector featuring the Higgs as a pseudo NambuGoldstone boson (pNGB) arising from the symmetry breaking $S O(5) \rightarrow S O(4)$, known as the Minimal Composite Higgs model (MCHM) [81,82]. The quark fields are embedded in $\mathbf{5}_{L}^{u}, \mathbf{1}_{R}^{u}, \mathbf{5}_{L}^{d}, \mathbf{1}_{R}^{d}$ representations of $S O(5)$, while all lepton fields are embedded in only two representations, $\mathbf{5}_{L}^{\ell}, \mathbf{1 4}_{R}^{\ell}$, per generation. As mentioned before, we can explain the tiny neutrino masses via a type-III seesaw mechanism, since the symmetric representation $\mathbf{1 4}_{R}^{\ell} \cong(\mathbf{1}, \mathbf{1})+(\mathbf{2}, \mathbf{2})+(\mathbf{3}, \mathbf{3})$ of $S O(5) \cong S U(2)_{L} \times S U(2)_{R}$ can host both an electrically charged $S U(2)_{L}$ singlet lepton $\left(\ell_{R}\right)$ and a heavy fermionic seesaw triplet of $S U(2)_{L}$. This unification of right handed leptons comes along with a more minimal quark representation than in known models, because the enhanced leptonic contribution to the Higgs potential, originating from the symmetric $S O(5)$ representation, allows for a viable electroweak symmetry breaking with all right handed SM-quarks inert under $S O(5)$ and the left-handed ones in the fundamental (see [21,22] for details). Thus, the model features less degrees of freedom than standard incarnations, such as the $\mathrm{MCHM}_{5}$.

One of the main challenges of all these scenarios featuring composite leptons is the generation of dangerous FCNCs through the exchange of the very same vector resonances producing the violation of LFU. Since, in general, they will also contribute to extremely well measured lepton flavor violating processes like $\mu \rightarrow e \gamma, \tau \rightarrow \mu \gamma, \mu \rightarrow e e e$, and $\mu-e$ conversion, they typically require the addition of some nontrivial flavor symmetry. In the model at hand, it turns out that the reduced number of composite operators mixing with the light leptons naturally allows for a very strong flavor protection along the lines of minimal flavor violation, since a single spurion allows to break the flavor symmetry [21]. Regarding the quark sector, in the model at hand the left handed current $\bar{s}_{L} \gamma^{\mu} b_{L}$ (i.e., $X=L$ ) will in general dominate, with the compositeness of $b_{L}$ following from the large top mass, but also non-negligible contributions from right-handed quarks are possible.

Let us conclude this discussion by stressing that if the effect of the model would be mostly due to the muon instead of the electron, neither the $R_{K^{*}}$ nor the $R_{K}$ anomaly could be addressed. Once the effect originates from a right-handed lepton current, it is required that the electron channel dominates in order to resolve the anomalies, see Fig. 1. On the other hand, contrary to the case of NP mostly in the muon current, in this case both chiralities for the quark current are fine. Thus, the very peculiar pattern of effects in our composite lepton scenario fits very well with experimental observation, predicting

$R_{K} \sim R_{K^{*}}<1$. 


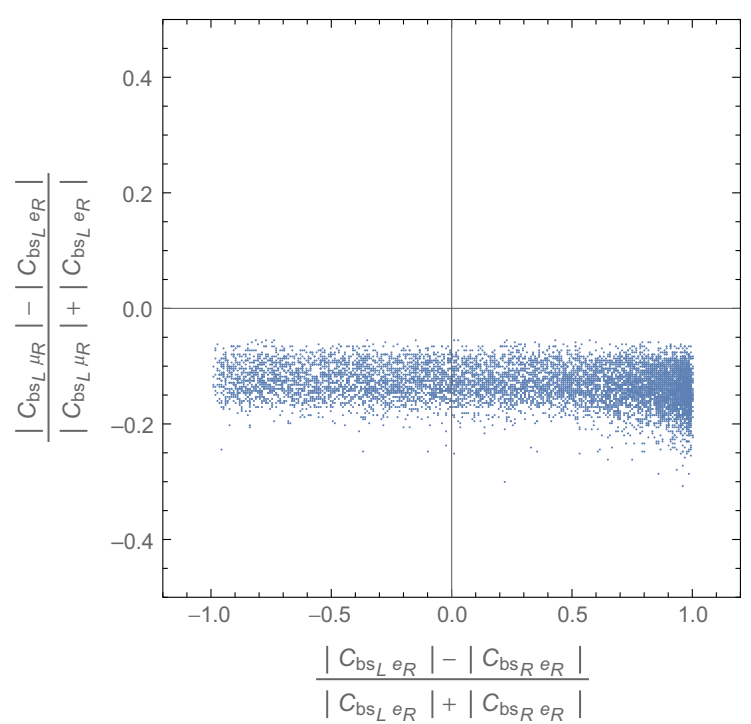

Fig. 2 Pattern of coefficients generated in the composite model. See text for details

Before presenting our final numerical results for these observables, we show in Fig. 2 the pattern of Wilson coefficients generated in the composite Higgs model. Here, and in the following, the points shown correspond to a scan over the parameter space of a 5D gauge-Higgs-unification description of the composite Higgs framework detailed above. Brane and bulk masses have been generated randomly (see [21] for more details), requiring the correct SM spectrum to emerge at low energies, while the pNGB decay constant has been chosen as $f=1.2 \mathrm{TeV}$, such as to solve the hierarchy problem while being in agreement with electroweak precision data. The large density of points in the lower right quadrant confirms numerically the expectation lined out before of $\mathcal{O}_{b s_{L} \ell_{R}}$ dominating in general, while in some regions of parameter space also $\mathcal{O}_{b s_{R} \ell_{R}}$ can be significant, all leading to the pattern (20).

After this general discussion, we present our predictions in the $R_{K^{*}}$ vs. $R_{K}$ plane in the left panel of Fig. 3. One can clearly observe the sought pattern of $R_{K} \sim R_{K^{*}}<1$, emerging as discussed in detail above. It is worth mentioning that points with SM-like values of $R_{K}$ and $R_{K^{*}}$ correspond to points of the scan with small FCNCs in the quark sector, since violation of LFU is always present in the model. This can be seen in particular in Fig. 2, since the points never reach the $(y=0)$-axis. As explained above, this is due to the fact that different values of $\epsilon_{\ell_{R}}, 0 \ll \epsilon_{\tau_{R}} \ll \epsilon_{\mu_{R}} \ll \epsilon_{e_{R}}$, are always needed in order to reproduce the spectrum in the lepton sector. Given that the masses of the vector resonances mediating these processes are fixed by $f$ to be relatively high, and that one needs to overcome the absence of interference with the SM contribution, agreement with the experimental values of $R_{K}$ and $R_{K} *$ requires points with rather sizable
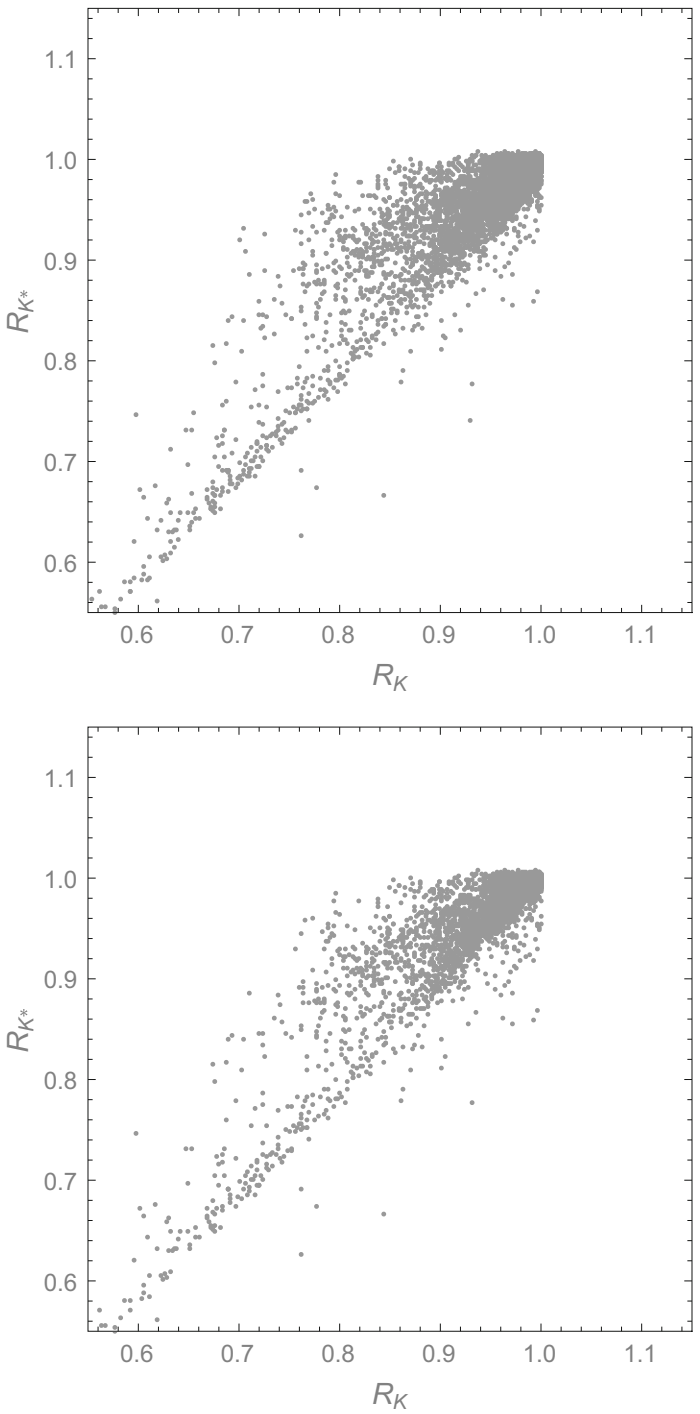

Fig. 3 Predictions in the $R_{K}-R_{K^{*}}$ plane. In the lower plot, points that feature a tension with the constraints from $B_{s} \rightarrow \mu^{+} \mu^{-}$of more than $1 \sigma$ are rejected. See text for details

FCNCs, which can be obtained in the considered parameter space.

Since along with $\mathcal{O}_{b s_{X} e_{R}}$ also, subleadingly, $\mathcal{O}_{b s_{X} \mu_{R}}$ is generated, all the points need to face constraints from the rather well-measured branching ratio $\mathcal{B}\left(B_{s} \rightarrow \mu^{+} \mu^{-}\right)$, which, in the chiral basis, is given by $[83]^{14}$

$$
\begin{aligned}
& \frac{\mathcal{B}\left(B_{s} \rightarrow \ell^{+} \ell^{-}\right)}{\mathcal{B}\left(B_{s} \rightarrow \ell^{+} \ell^{-}\right)_{\mathrm{SM}}} \\
& =\left|1+\frac{C_{b s_{L} \ell_{R}}^{\mathrm{NP}}-C_{b s_{L} \ell_{L}}^{\mathrm{NP}}-C_{b s_{R} \ell_{R}}^{\mathrm{NP}}+C_{b s_{R} \ell_{L}}^{\mathrm{NP}}}{C_{b s_{L} \ell_{R}}^{\mathrm{SM}}-C_{b s_{L} \ell_{L}}^{\mathrm{SM}}}\right|^{2},
\end{aligned}
$$

\footnotetext{
${ }^{14}$ Here, we neglect scalar and pseudoscalar operators, not generated in the model at hand to good approximation.
} 


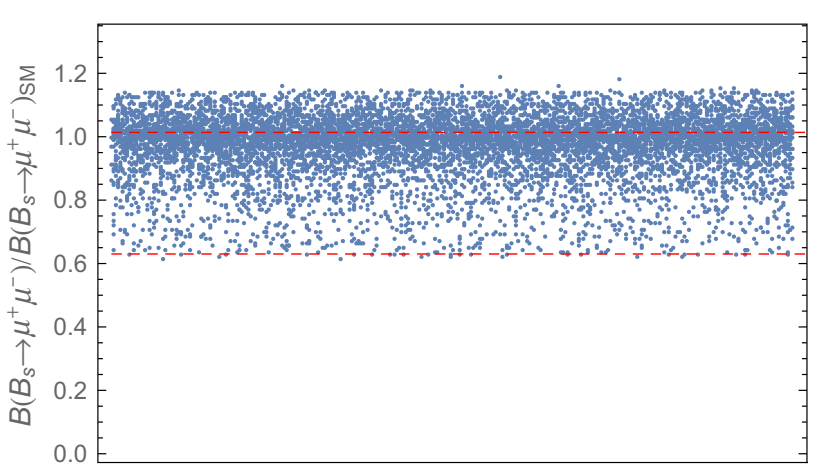

Fig. 4 Predictions for $\mathcal{B}\left(B_{s} \rightarrow \mu^{+} \mu^{-}\right) / \mathcal{B}\left(B_{s} \rightarrow \mu^{+} \mu^{-}\right)_{\mathrm{SM}}$ in the composite Higgs scenario. The experimental $1 \sigma$ range is depicted by the red dashed lines

where [84-86] (see also [36])

$$
\begin{aligned}
& \mathcal{B}\left(B_{s} \rightarrow \mu^{+} \mu^{-}\right)_{\mathrm{SM}}=(3.65 \pm 0.23) \times 10^{-9}, \\
& \mathcal{B}\left(B_{s} \rightarrow \mu^{+} \mu^{-}\right)_{\exp }=\left(3.0 \pm 0.6_{-0.2}^{+0.3}\right) \times 10^{-9}, \\
& \mathcal{B}\left(B_{s} \rightarrow e^{+} e^{-}\right)_{\mathrm{SM}}=(8.54 \pm 0.55) \times 10^{-14}, \\
& \mathcal{B}\left(B_{S} \rightarrow e^{+} e^{-}\right)_{\exp }<2.8 \times 10^{-7} @ 90 \% \mathrm{CL} .
\end{aligned}
$$

Note that in general the muonic final states are more stringently constrained experimentally, leaving more room for effects in the electron channel, which does not constrain the model at hand at all. The former, on the other hand, has a (mild) impact on the setup. In general, avoiding effects in $B_{s} \rightarrow \ell^{+} \ell^{-}$, requires either a leptonic or a quark vectorcurrent - in fact the decay tests products of axial-vector currents. In consequence, the negative $C_{b s_{L} \mu_{L}}^{\mathrm{NP}}$ solution to the $R_{K^{(*)}}$ anomalies could be accompanied by a $C_{b s_{R} \mu_{L}}^{\mathrm{NP}}$ contribution, to cancel effects in $B_{S} \rightarrow \ell^{+} \ell^{-}$, which would however go in the wrong direction with respect to $R_{K^{*}}$. The second option is an addition of $C_{b s_{L} \mu_{R}}^{\mathrm{NP}}$, which is less problematic since suppressed by the smaller interference with the SM, and corresponds to the $C_{9}$ solution of the $b \rightarrow s \ell^{+} \ell^{-}$anomalies. Note that to go into the direction of the very modest (negative) deviation from the SM prediction in $B_{s} \rightarrow \mu^{+} \mu^{-}$, for a fixed chirality for one fermion current, the other should feature the larger effect in the opposite chirality (for positive Wilson coefficients), i.e., $C_{b s_{X} \mu_{Y}}^{\mathrm{NP}}-C_{b s_{X} \mu_{X}}^{\mathrm{NP}}>0$ or $C_{b s_{Y} \mu_{X}}^{\mathrm{NP}}-C_{b s_{X} \mu_{X}}^{\mathrm{NP}}>0,(X, Y) \in\{(L, R),(R, L)\}$ (see Eq. (21)). This means in particular that an effect stemming solely from a negative $C_{b s_{L} \mu_{L}}^{\mathrm{NP}}$ goes in principle in a favorable direction.

For the composite Higgs model considered, effects are coming from non-vanishing $C_{b s_{L-R} \mu_{R}}^{\mathrm{NP}}$, but are modest in general, as can be seen from Fig. 4 where we show the ratio $\mathcal{B}\left(B_{S} \rightarrow \mu^{+} \mu^{-}\right) / \mathcal{B}\left(B_{s} \rightarrow \mu^{+} \mu^{-}\right)_{\mathrm{SM}}$, together with the experimental $1 \sigma$ range, the latter depicted by the red dashed lines. Most of the points lie within the corresponding range (while basically all meet the $2 \sigma$ constraints). The impact of the $1 \sigma$ bound on the composite solution to the $R_{K^{(*)}}$ anomalies is visualized in the right panel of Fig. 3, where we reject the parameter-space points that do not meet this constraint. As can be seen, the effect is very modest.

We now move to study the correlation with $B_{S}-\bar{B}_{S}$ mixing, focusing on the mass difference between the heavy and light mass eigenstate $\Delta M_{B_{s}} \equiv M_{H}^{s}-M_{L}^{s}$ (see e.g. [87]). The relevant additional operators, entering $B_{S}-\bar{B}_{S}$ mixing, are contained in the $\Delta B=2$ Lagrangian [46]

$\mathcal{H}_{\text {eff }}^{\Delta B=2}=-\sum_{X Y=L L, L R, R R} C_{V}^{X Y} \mathcal{O}_{V}^{X Y}+$ h.c.,

where

$\mathcal{O}_{V}^{X Y}=\left(\bar{s} \gamma_{\mu} P_{X} b\right)\left(\bar{s} \gamma^{\mu} P_{Y} b\right)$,

and are generated predominantly via the exchange of gluon resonances in the composite Higgs framework. Note that we neglect again scalar (and tensor) operators, not present for the model at hand to good approximation. Our general prediction, obtained via a fit to the numerical results [46] at the scale $\mu=2 \mathrm{TeV}$, reads

$$
\begin{aligned}
& \frac{\Delta M_{B_{s}}}{\Delta M_{B_{s}}^{\mathrm{SM}}} \simeq 1+\left(35,380 \operatorname{Re} C_{V}^{L R \mathrm{NP}}\right. \\
& \left.\quad-10,530 \operatorname{Re}\left[C_{V}^{L L \mathrm{NP}}+C_{V}^{R R \mathrm{NP}}\right]\right) \mathrm{TeV}^{2},
\end{aligned}
$$

where $\Delta M_{B_{s}}^{\mathrm{SM}}=1.313 \times 10^{-11} \mathrm{GeV}$.

In Fig. 5 we provide again the results in the $R_{K^{*}}$ vs. $R_{K}$ plane, where now the color code depicts the size of the corrections to the SM value of $\Delta M_{B_{s}}$, with light (dark) blue corresponding to modest (sizable) modifications. We find that there is a considerable amount of points with (20-30) \% effects in $R_{K^{(*)}}$ and deviations in $\Delta M_{B_{s}}$ at the $\lesssim 20 \%$ level, which is in the ballpark of the uncertainty in the SM prediction. In consequence, values $R_{K} \sim R_{K^{*}} \sim(0.7-0.8)$ are possible, while constraints from $B_{s}-\bar{B}_{s}$ mixing are met. One should note that, quite generally, the size of quark FCNCs required to obtain large deviations in $R_{K}$ and $R_{K^{*}}$ leads to large modifications in $\Delta M_{B_{s}}$. However, small deviations in $\Delta M_{B_{s}}$ can be achieved via a cancellation between LH and $\mathrm{RH}$ quark currents, so the presence of both $C_{b s_{L} \ell_{X}}^{\mathrm{NP}}$ and $C_{b s_{R} \ell_{X}}^{\mathrm{NP}}$ is somewhat preferred, moving away from the $R_{K}=R_{K^{*}}$ line (see Fig. 1). This, which may seem like a trivial fact, makes a large difference when compared to the rest of $Z^{\prime}$ explanations of these anomalies. The reason is that, only for the case studied here of NP mostly in RH electrons, one can have an effect in both $\bar{b}_{R} \gamma^{\mu} s_{R}$ and $\bar{b}_{L} \gamma^{\mu} s_{L}$ and still get agreement with both $R_{K}$ and $R_{K^{*}}$ (as discussed in the previous section). This means in particular that, if the prediction for $\Delta M_{B_{s}}$ quoted by [88] is taken at face value, accepting some accidental cancellations, the scenario considered here 


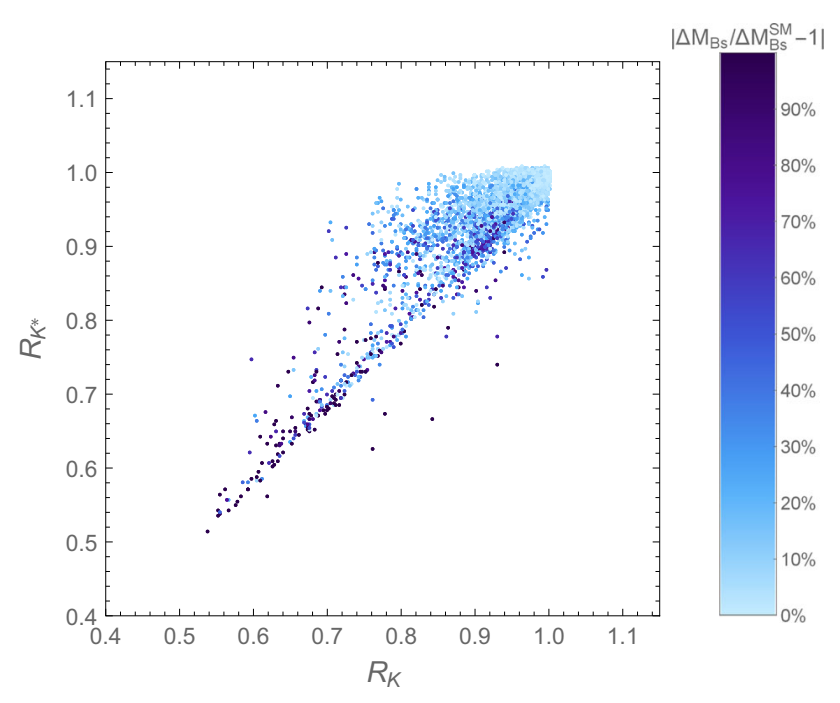

Fig. 5 Predictions in the $R_{K}-R_{K^{*}}$ plane, where the colors depict the size of the corrections to $\Delta M_{B_{s}}$. See text for details

would provide the only allowed $Z^{\prime}$ model accommodating both anomalies. ${ }^{15}$

Finally, another interesting set of observables in the field of semi-leptonic $B$ decays are the angular dependencies of the $B \rightarrow K^{*} \mu^{+} \mu^{-}$decay rate. Of particular interest is the coefficient $P_{5}^{\prime}$, which belongs to a class of observables constructed such as to cancel hadronic uncertainties [23-31], which shows an interesting deviation with respect to the SM prediction, see e.g., $[8,16]$. Our results for $P_{5}^{\prime}$ in the $q^{2} \in[4.3,8.68] \mathrm{GeV}^{2}$ bin are given in Fig. 6, where we plot the correlation between $P_{5}^{\prime}$ and $R_{K}$, visualizing again the size of the corrections to $\Delta M_{B_{s}}$ as different shades of blue. The SM prediction $P_{5[4.3,8.68]}^{\prime} \approx-0.8$ and the experimentally preferred value $P_{5[4.3,8.68]}^{\prime} \approx-0.2[8,16]$ are given as red and green dashed lines, respectively. From this final plot it is evident that the proposed composite model can address the $R_{K}$ and $R_{K^{*}}$ anomalies, being in agreement with $B_{s}-\bar{B}_{s}$ mixing and $\mathcal{B}\left(B_{s} \rightarrow \ell^{+} \ell^{-}\right)$, while also the fit to the $P_{5}^{\prime}$ results can be improved (via the light-blue points approaching $P_{5[4.3,8.68]}^{\prime} \approx-0.2$ and featuring $R_{K} \sim(0.7-0.8)$ ). It thus furnishes an interesting setup both with respect to the gauge hierarchy problem - avoiding light top partners - and concerning the current pattern of experimental results in flavor physics.

Before concluding, let us mention further ways to test the proposed composite Higgs scenario. First, finding non-

\footnotetext{
15 Note that solutions to the $R_{K}$ anomaly are also subject to competitive constraints from searches for tails in the high $p_{T}$ di-lepton spectrum [89]. However, due to the suppressed couplings to light quarks, our setup avoids these bounds. Moreover, di-lepton searches will be less effective, since one typically expects the neutral vector mediators to decay via a lepton partner, leading to different decay topologies [90], see also below.
}

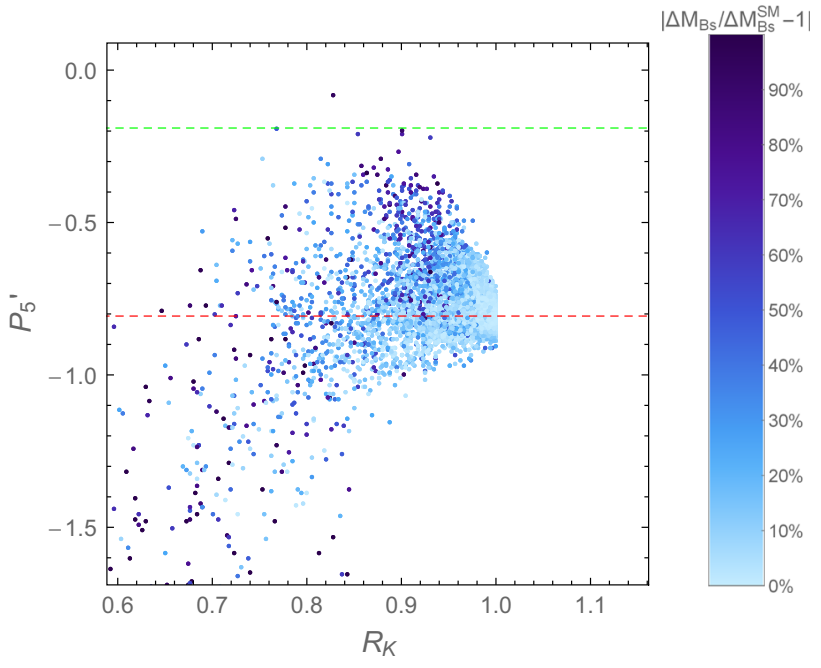

Fig. 6 Correlation between $P_{5}^{\prime}$ and $R_{K}$, where the colors depict the size of the corrections to $\Delta M_{B_{S}}$. See text for details

vanishing coefficients of four-lepton operators at future lepton colliders (in particular of type $\left(\bar{e}_{R} \gamma^{\mu} e_{R}\right)\left(\bar{e}_{R} \gamma_{\mu} e_{R}\right)$ $[21,80])$ could be interpreted as a hint for lepton compositeness. Moreover, along the line of 'conventional' light top partners, the model at hand contains leptonic resonances $\ell^{\prime}$ somewhat below the typical resonance scale, $m_{\ell^{\prime}}<m_{Z^{\prime}}$ [22], which could be searched for directly. Finally, due to the very fact that these particles can be lighter than the $Z^{\prime}$, new decay channels such as $Z^{\prime} \rightarrow \ell^{\prime} \ell$ open up, which have been investigated in [90], and would be a smoking gun for the setup.

\section{Conclusions}

We have scrutinized hints for lepton flavor universality violation in $B$-meson decays, focusing first on the general properties of the anomalies in an EFT approach. Here, we emphasized a simultaneous solution to the $R_{K}$ and $R_{K^{*}}$ anomalies via effects in right-handed lepton currents, not worked out before. We stressed that this solution requires the dominant contribution originating from electron (and not muon) currents and presented a composite Higgs scenario, where this pattern emerges in a natural way. In fact, the model provides one of the very few scenarios, that features all ingredients to consistently resolve the anomalies, without being actually constructed for that purpose: it predicts LFU violation and sizable FCNCs involving the third quark generation, features a strong protection from FCNCs in the lepton sector, and allows for the absence of ultra-light top partners at the LHC. We also discussed the impact of operators addressing the $R_{K^{(*)}}$ measurements on other flavor observables, such as $\mathcal{B}\left(B_{s} \rightarrow \ell^{+} \ell^{-}\right), \Delta M_{B_{s}}$, and $P_{5}^{\prime}$. For the explicit model at 
hand, we found that all constraints are met, while it is possible to simultaneously resolve the (more controversial) $P_{5}^{\prime}$ anomaly.

Acknowledgements We are grateful to Marco Nardecchia, David Straub and Jorge Martin Camalich, for useful comments and discussions. FG thanks the CERN Theory Department, where part of this work was performed, for its hospitality. The research of AC was supported by a Marie Skłodowska-Curie Individual Fellowship of the European Community's Horizon 2020 Framework Programme for Research and Innovation under contract number 659239 (NP4theLHC14) and by the Cluster of Excellence Precision Physics, Fundamental Interactions and Structure of Matter (PRISMA-EXC 1098) and Grant 05H12UME of the German Federal Ministry for Education and Research (BMBF).

Open Access This article is distributed under the terms of the Creative Commons Attribution 4.0 International License (http://creativecomm ons.org/licenses/by/4.0/), which permits unrestricted use, distribution, and reproduction in any medium, provided you give appropriate credit to the original author(s) and the source, provide a link to the Creative Commons license, and indicate if changes were made. Funded by SCOAP ${ }^{3}$.

\section{References}

1. R. Aaij et al., Phys. Rev. Lett. 111, 191801 (2013). https://doi.org/ 10.1103/PhysRevLett.111.191801

2. R. Aaij et al., JHEP 02, 104 (2016). https://doi.org/10.1007/ JHEP02(2016) 104

3. S. Wehle et al., Phys. Rev. Lett. 118(11), 111801 (2017). https:// doi.org/10.1103/PhysRevLett.118.111801

4. CMS Collaboration, Measurement of the $P_{1}$ and $P_{5}^{\prime}$ angular parameters of the decay $\mathrm{B}^{0} \rightarrow \mathrm{K}^{* 0} \mu^{+} \mu^{-}$in proton-proton collisions at $\sqrt{s}=8 \mathrm{TeV}$. CMS-PAS-BPH-15-008 (2017)

5. ATLAS Collaboration, Angular analysis of $B_{d}^{0} \rightarrow K^{*} \mu^{+} \mu^{-}$ decays in $p p$ collisions at $\sqrt{s}=8 \mathrm{TeV}$ with the ATLAS detector. ATLAS-CONF-2017-023 (2017)

6. R. Aaij et al., JHEP 06, 133 (2014). https://doi.org/10.1007/ JHEP06(2014) 133

7. R. Aaij et al., JHEP 09, 179 (2015). https://doi.org/10.1007/ JHEP09(2015) 179

8. S. Descotes-Genon, J. Matias, J. Virto, Phys. Rev. D 88, 074002 (2013). https://doi.org/10.1103/PhysRevD.88.074002

9. W. Altmannshofer, D.M. Straub, Eur. Phys. J. C 73, 2646 (2013). https://doi.org/10.1140/epjc/s10052-013-2646-9

10. F. Beaujean, C. Bobeth, D. van Dyk, Eur. Phys. J. C 74, 2897 (2014). https://doi.org/10.1140/epjc/s10052-014-2897-0 [Erratum: Eur. Phys. J. C 74, 3179 (2014). https://doi.org/10.1140/epjc/ s10052-014-3179-6]

11. T. Hurth, F. Mahmoudi, JHEP 04, 097 (2014). https://doi.org/10. 1007/JHEP04(2014)097

12. R. Gauld, F. Goertz, U. Haisch, Phys. Rev. D 89, 015005 (2014). https://doi.org/10.1103/PhysRevD.89.015005

13. W. Altmannshofer, D.M. Straub, Eur. Phys. J. C 75(8), 382 (2015). https://doi.org/10.1140/epjc/s10052-015-3602-7

14. S. Descotes-Genon, L. Hofer, J. Matias, J. Virto, JHEP 06, 092 (2016). https://doi.org/10.1007/JHEP06(2016)092

15. T. Hurth, F. Mahmoudi, S. Neshatpour, Nucl. Phys. B 909, 737 (2016). https://doi.org/10.1016/j.nuclphysb.2016.05.022

16. W. Altmannshofer, C. Niehoff, P. Stangl, D.M. Straub, Eur. Phys. J. C 77(6), 377 (2017). https://doi.org/10.1140/epjc/ s10052-017-4952-0
17. G. Hiller, F. Kruger, Phys. Rev. D 69, 074020 (2004). https://doi. org/10.1103/PhysRevD.69.074020

18. R. Aaij et al., Phys. Rev. Lett. 113, 151601 (2014). https://doi.org/ 10.1103/PhysRevLett.113.151601

19. M. Bordone, G. Isidori, A. Pattori, Eur. Phys. J. C 76(8), 440 (2016). https://doi.org/10.1140/epjc/s10052-016-4274-7

20. R. Aaij et al., JHEP 08, 055 (2017). https://doi.org/10.1007/ JHEP08(2017)055

21. A. Carmona, F. Goertz, Phys. Rev. Lett. 116(25), 251801 (2016). https://doi.org/10.1103/PhysRevLett.116.251801

22. A. Carmona, F. Goertz, JHEP 05, 002 (2015). https://doi.org/10. 1007/JHEP05(2015)002

23. J. Matias, F. Mescia, M. Ramon, J. Virto, JHEP 04, 104 (2012). https://doi.org/10.1007/JHEP04(2012)104

24. S. Jäger, J. Martin Camalich, JHEP 05, 043 (2013). https://doi.org/ 10.1007/JHEP05(2013)043

25. S. Descotes-Genon, T. Hurth, J. Matias, J. Virto, JHEP 05, 137 (2013). https://doi.org/10.1007/JHEP05(2013)137

26. J. Lyon, R. Zwicky, Resonances gone topsy turvy-the charm of QCD or new physics in $b \rightarrow s \ell^{+} \ell^{-}$? Report no. EDINBURGH-14-10, CP3-ORIGINS-2014-021-DNRF90, DIAS2014-21 (2014). arXiv:1406.0566

27. S. Descotes-Genon, L. Hofer, J. Matias, J. Virto, JHEP 12, 125 (2014). https://doi.org/10.1007/JHEP12(2014)125

28. S. Jäger, J. Martin Camalich, Phys. Rev. D 93(1), 014028 (2016). https://doi.org/10.1103/PhysRevD.93.014028

29. M. Ciuchini, M. Fedele, E. Franco, S. Mishima, A. Paul, L. Silvestrini, M. Valli, JHEP 06, 116 (2016). https://doi.org/10.1007/ JHEP06(2016)116

30. B. Capdevila, S. Descotes-Genon, L. Hofer, J. Matias, JHEP 04, 016 (2017). https://doi.org/10.1007/JHEP04(2017)016

31. V.G. Chobanova, T. Hurth, F. Mahmoudi, D. Martinez Santos, S. Neshatpour, JHEP 07, 025 (2017). https://doi.org/10.1007/ JHEP07(2017)025

32. B. Grinstein, M.J. Savage, M.B. Wise, Nucl. Phys. B 319, 271 (1989). https://doi.org/10.1016/0550-3213(89)90078-3

33. G. Buchalla, A.J. Buras, M.E. Lautenbacher, Rev. Mod. Phys. 68, 1125 (1996). https://doi.org/10.1103/RevModPhys.68.1125

34. G. Hiller, M. Schmaltz, Phys. Rev. D 90, 054014 (2014). https:// doi.org/10.1103/PhysRevD.90.054014

35. F. Beaujean, C. Bobeth, S. Jahn, Eur. Phys. J. C 75(9), 456 (2015). https://doi.org/10.1140/epjc/s10052-015-3676-2

36. R. Fleischer, R. Jaarsma, G. Tetlalmatzi-Xolocotzi, JHEP 05, 156 (2017). https://doi.org/10.1007/JHEP05(2017)156

37. R. Alonso, B. Grinstein, J. Martin Camalich, Phys. Rev. Lett. 113, 241802 (2014). https://doi.org/10.1103/PhysRevLett.113.241802

38. B. Capdevila, S. Descotes-Genon, L. Hofer, J. Matias, J. Virto, PoS LHCP2016, 073 (2016)

39. G. Hiller, M. Schmaltz, JHEP 02, 055 (2015). https://doi.org/10. 1007/JHEP02(2015)055

40. G. D'Amico, M. Nardecchia, P. Panci, F. Sannino, A. Strumia, R. Torre, A. Urbano, JHEP 09, 010 (2017). https://doi.org/10.1007/ JHEP09(2017)010

41. A. Ali, P. Ball, L.T. Handoko, G. Hiller, Phys. Rev. D 61, 074024 (2000). https://doi.org/10.1103/PhysRevD.61.074024

42. C. Bouchard, G.P. Lepage, C. Monahan, H. Na, J. Shigemitsu, Phys. Rev. D 88(5), 054509 (2013). https://doi.org/10.1103/PhysRevD. 88.079901 [Erratum: Phys. Rev. D 88(7), 079901 (2013). https:// doi.org/10.1103/PhysRevD.88.054509]

43. A.J. Buras, J. Girrbach-Noe, C. Niehoff, D.M. Straub, JHEP 02, 184 (2015). https://doi.org/10.1007/JHEP02(2015)184

44. A. Paul, D.M. Straub, JHEP 04, 027 (2017). https://doi.org/10. 1007/JHEP04(2017)027

45. R. Contino, A. Falkowski, F. Goertz, C. Grojean, F. Riva, JHEP 07, 144 (2016). https://doi.org/10.1007/JHEP07(2016)144 
46. D. Straub, P. Stangl, C. Niehoff, E. Gurler, W. Zeren, J. Kumar, S. Reicher, F. Beaujean, flav-io/flavio v0.21.1 (2017). https://doi.org/ 10.5281/zenodo.569011

47. C. Bobeth, G. Hiller, G. Piranishvili, JHEP 07, 106 (2008). https:// doi.org/10.1088/1126-6708/2008/07/106

48. C. Hambrock, G. Hiller, S. Schacht, R. Zwicky, Phys. Rev. D 89(7), 074014 (2014). https://doi.org/10.1103/PhysRevD.89.074014

49. A. Bharucha, D.M. Straub, R. Zwicky, JHEP 08, 098 (2016). https://doi.org/10.1007/JHEP08(2016)098

50. A. Crivellin, G. D'Ambrosio, J. Heeck, Phys. Rev. Lett. 114, 151801 (2015). https://doi.org/10.1103/PhysRevLett.114.151801

51. A. Crivellin, G. D’Ambrosio, J. Heeck, Phys. Rev. D 91(7), 075006 (2015). https://doi.org/10.1103/PhysRevD.91.075006

52. C. Niehoff, P. Stangl, D.M. Straub, Phys. Lett. B 747, 182 (2015). https://doi.org/10.1016/j.physletb.2015.05.063

53. D. Aristizabal Sierra, F. Staub, A. Vicente, Phys. Rev. D 92(1), 015001 (2015). https://doi.org/10.1103/PhysRevD.92.015001

54. A. Celis, J. Fuentes-Martin, M. Jung, H. Serodio, Phys. Rev. D 92(1), 015007 (2015). https://doi.org/10.1103/PhysRevD.92. 015007

55. A. Greljo, G. Isidori, D. Marzocca, JHEP 07, 142 (2015). https:// doi.org/10.1007/JHEP07(2015)142

56. A. Falkowski, M. Nardecchia, R. Ziegler, JHEP 11, 173 (2015). https://doi.org/10.1007/JHEP11(2015)173

57. B. Allanach, F.S. Queiroz, A. Strumia, S. Sun, Phys. Rev. D 93(5), 055045 (2016). https://doi.org/10.1103/PhysRevD.93. 055045 [Erratum: Phys. Rev. D 95(11), 119902 (2017). https:// doi.org/10.1103/PhysRevD.95.119902]

58. C.W. Chiang, X.G. He, G. Valencia, Phys. Rev. D 93(7), 074003 (2016). https://doi.org/10.1103/PhysRevD.93.074003

59. S.M. Boucenna, A. Celis, J. Fuentes-Martin, A. Vicente, J. Virto, Phys. Lett. B 760, 214 (2016). https://doi.org/10.1016/j.physletb. 2016.06.067

60. E. Megias, G. Panico, O. Pujolas, M. Quiros, JHEP 09, 118 (2016). https://doi.org/10.1007/JHEP09(2016)118

61. W. Altmannshofer, S. Gori, S. Profumo, F.S. Queiroz, JHEP 12, 106 (2016). https://doi.org/10.1007/JHEP12(2016)106

62. A. Crivellin, J. Fuentes-Martin, A. Greljo, G. Isidori, Phys. Lett. B 766, 77 (2017). https://doi.org/10.1016/j.physletb.2016.12.057

63. I. Garcia Garcia, JHEP 03, 040 (2017). https://doi.org/10.1007/ JHEP03(2017)040

64. D. Bhatia, S. Chakraborty, A. Dighe, JHEP 03, 117 (2017). https:// doi.org/10.1007/JHEP03(2017)117

65. C. Bonilla, T. Modak, R. Srivastava, J.W.R. Valle, $U(1)_{B_{3}-3 L_{\mu}}$ gauge symmetry as a simple description of $b \rightarrow s$ anomalies. Phys. Rev. D 98(9), 095002 (2018). https://doi.org/10.1103/PhysRevD. 98.095002

66. R. Alonso, P. Cox, C. Han, T.T. Yanagida, Phys. Lett. B 774, 643 (2017). https://doi.org/10.1016/j.physletb.2017.10.027

67. J. Ellis, M. Fairbairn, P. Tunney, Anomaly-free models for flavour anomalies. Eur. Phys. J. C 78(3), 238 (2018). https://doi.org/10. 1140/epjc/s10052-018-5725-0. arXiv:1705.03447

68. Y. Tang, Y.-L. Wu, Flavor non-universal gauge interactions and anomalies in B-meson decays. Chin. Phys. C 42(3), 033104 (2018). https://doi.org/10.1088/1674-1137/42/3/033104. arXiv: 1705.05643
69. C.-W. Chiang, X.-G. He, J. Tandean, X.-B. Yuan, $R_{K^{(*)}}$ and related $b \rightarrow s \ell \bar{\ell}$ anomalies in minimal flavor violation framework with $Z^{\prime}$ boson. Phys. Rev. D96(11), 115022. https://doi.org/10.1103/ PhysRevD.96.115022. arXiv:1706.02696

70. S.F. King, JHEP 08, 019 (2017). https://doi.org/10.1007/ JHEP08(2017)019

71. D. Buttazzo, A. Greljo, G. Isidori, D. Marzocca, JHEP 11, 044 (2017). https://doi.org/10.1007/JHEP11(2017)044

72. E. Megias, M. Quiros, L. Salas, Phys. Rev. D 96(7), 075030 (2017). https://doi.org/10.1103/PhysRevD.96.075030

73. J.M. Cline, J. Martin Camalich, Phys. Rev. D 96(5), 055036 (2017). https://doi.org/10.1103/PhysRevD.96.055036

74. J.M. Cline, Phys. Rev. D 97(1), 015013 (2018). https://doi.org/10. 1103/PhysRevD.97.015013

75. G. Ballesteros, A. Carmona, M. Chala, Eur. Phys. J. C 77(7), 468 (2017). https://doi.org/10.1140/epjc/s10052-017-5040-1

76. M. Chala, JHEP 01, 122 (2013). https://doi.org/10.1007/ JHEP01(2013)122

77. M. Carena, A.D. Medina, N.R. Shah, C.E.M. Wagner, Phys. Rev. D 79, 096010 (2009). https://doi.org/10.1103/PhysRevD.79.096010

78. F. del Aguila, A. Carmona, J. Santiago, JHEP 08, 127 (2010). https://doi.org/10.1007/JHEP08(2010)127

79. C. Hagedorn, M. Serone, JHEP 02, 077 (2012). https://doi.org/10. 1007/JHEP02(2012)077

80. A. Carmona, F. Goertz, Nucl. Part. Phys. Proc. 285-286, 93 (2017). https://doi.org/10.1016/j.nuclphysbps.2017.03.017

81. R. Contino, Y. Nomura, A. Pomarol, Nucl. Phys. B 671, 148 (2003). https://doi.org/10.1016/j.nuclphysb.2003.08.027

82. K. Agashe, R. Contino, A. Pomarol, Nucl. Phys. B 719, 165 (2005). https://doi.org/10.1016/j.nuclphysb.2005.04.035

83. K. De Bruyn, R. Fleischer, R. Knegjens, P. Koppenburg, M. Merk, A. Pellegrino, N. Tuning, Phys. Rev. Lett. 109, 041801 (2012). https://doi.org/10.1103/PhysRevLett.109.041801

84. C. Bobeth, M. Gorbahn, T. Hermann, M. Misiak, E. Stamou, M. Steinhauser, Phys. Rev. Lett. 112, 101801 (2014). https://doi.org/ 10.1103/PhysRevLett.112.101801

85. R. Aaij et al., Phys. Rev. Lett. 118(19), 191801 (2017). https://doi. org/10.1103/PhysRevLett.118.191801

86. T. Aaltonen et al., Phys. Rev. Lett. 102, 201801 (2009). https://doi. org/10.1103/PhysRevLett.102.201801

87. A.J. Buras, in Probing the standard model of particle interactions. Proceedings, Summer School in Theoretical Physics, NATO Advanced Study Institute, 68th Session, Les Houches, France, July 28-September 5, 1997. Pt. 1, 2 (1998), pp. 281-539

88. L. Di Luzio, M. Kirk, A. Lenz, Phys. Rev. D 97, 095035 (2018). https://doi.org/10.1103/PhysRevD.97.095035

89. A. Greljo, D. Marzocca, Eur. Phys. J. C 77(8), 548 (2017). https:// doi.org/10.1140/epjc/s10052-017-5119-8

90. M. Chala, M. Spannowsky, Phys. Rev. D 98(3), 035010 (2018). https://doi.org/10.1103/PhysRevD.98.035010 